\title{
Emergence of multi-drug resistant Salmonella Enteritidis strains harbouring novel virulence plasmid in Korea
}

\author{
Tae-Min La ${ }^{1}$, Taesoo Kim${ }^{1}$, Hong-Jae Lee ${ }^{1}$, Yoonsuk Lee ${ }^{1}$, Hyunjin Shin ${ }^{1}$, Yongjun Song ${ }^{1}$, \\ Gyuhee Ahn ${ }^{1}$, Hur Won ${ }^{1}$, Joong-Bok Lee ${ }^{2}$, Seung-Yong Park ${ }^{2}$, Chang-Seon Song ${ }^{1}$, In-Soo \\ $\mathrm{Choi}^{3}$, and Sang-Won Lee ${ }^{1}$ \\ ${ }^{1}$ Konkuk Univ \\ ${ }^{2}$ College of Veterinary Medicine, Konkuk University \\ ${ }^{3}$ Affiliation not available
}

September 11, 2020

\begin{abstract}
The Salmonella enterica subspecies serovar Enteritidis is the one of main serovars isolated from human patients with food poisoning and poultry without clinical signs. Consumption of poultry products contaminated with Salmonella Enteritidis is a common source of human salmonellosis. This study was aimed to determine the genetic relationships between Salmonella Enteritidis strains isolated from human patients and those isolated from poultry sources using whole-genome sequencing analysis. Seven of multi-drug resistant (MDR) strains of Salmonella Enteritidis were isolated from 234 retail chicken meats, internal organs of chicken, and straw bedding samples at chicken farms. Whole genome sequences of the MDR strains were determined using the Oxford Nanopore sequencing and compared with available whole genome sequences of the Salmonella Enteritidis strains previously isolated in Korea. Single-nucleotide polymorphism analysis of the whole genomes showed that all MDR Salmonella Enteritidis strains were genetically close and related to the FORC_019 strain isolated from human blood in 2015 in Korea. All of the MDR Salmonella Enteritidis strains contained a $110 \mathrm{~kb}$ of plasmid, and comparative plasmid analysis showed that all of the MDR Salmonella Enteritidis strains carried a novel fusion plasmid with genes coding virulence factors and antibiotic resistance proteins. The almost identical plasmid, with an extra 7.5-kb insertion sequence was also found in the FORC_019 strain. These results suggested that the single genetic lineage of MDR Salmonella Enteritidis that can cause salmonellosis in human is currently contaminating the Korean poultry industry.
\end{abstract}

Title: Emergence of multi-drug resistant SalmonellaEnteritidis strains harbouring novel virulence plasmid in Korea

Running title : Emergence of MDR SE having novel virulence plasmid

Authors: Tae-Min La, Taesoo Kim, Hong-Jae Lee, Yoonsuk Lee, Hyunjin Shin, Yongjun Song, Gyuhee Ahn, Won Hur, Joong-Bok Lee, Seung-Yong Park, Chang-Seon Song, In-Soo Choi, and Sang-Won Lee

College of Veterinary Medicine, Konkuk University, Seoul 05029, Republic of Korea

\section{Correspondence}

Sang-Won Lee, College of Veterinary Medicine, Konkuk University, Seoul 05029, Republic of Korea, phone +82-2-450-0445, fax +82-2-3437-1941, Email:odssey@konkuk.ac.kr

Keywords: whole-genome analysis, Salmonella Enteritidis, Multi-drug resistance, virulence plasmid, Salmonellosis

\section{Summary}


The Salmonella enterica subspecies serovar Enteritidis is the one of main serovars isolated from human patients with food poisoning and poultry without clinical signs. Consumption of poultry products contaminated with Salmonella Enteritidis is a common source of human salmonellosis. This study was aimed to determine the genetic relationships between Salmonella Enteritidis strains isolated from human patients and those isolated from poultry sources using whole-genome sequencing analysis.

Seven of multi-drug resistant (MDR) strains of SalmonellaEnteritidis were isolated from 234 retail chicken meats, internal organs of chicken, and straw bedding samples at chicken farms. Whole genome sequences of the MDR strains were determined using the Oxford Nanopore sequencing and compared with available whole genome sequences of theSalmonella Enteritidis strains previously isolated in Korea.

Single-nucleotide polymorphism analysis of the whole genomes showed that all MDR Salmonella Enteritidis strains were genetically close and related to the FORC_019 strain isolated from human blood in 2015 in Korea. All of the MDR Salmonella Enteritidis strains contained a $110 \mathrm{~kb}$ of plasmid, and comparative plasmid analysis showed that all of the MDR Salmonella Enteritidis strains carried a novel fusion plasmid with genes coding virulence factors and antibiotic resistance proteins. The almost identical plasmid, with an extra 7.5-kb insertion sequence was also found in the FORC_019 strain.

These results suggested that the single genetic lineage of MDRSalmonella Enteritidis that can cause salmonellosis in human is currently contaminating the Korean poultry industry.

\section{Introduction}

Non-typhoidal Salmonella enterica causes food-borne salmonellosis and has become a global health threat - The Salmonella entericaserovar Enteritidis is frequently isolated from human patients with salmonellosis caused by consumption of contaminated chicken meat and chicken products such as eggs . Epidemiological sources ofSalmonella outbreaks have been investigated using pulsed-field gel electrophoresis (PFGE) and multiple locus variable-number tandem repeats (MLVA) analysis. In previous studies, PFGE and MLVA analysis successfully detected the genetic relationship between SalmonellaEnteritidis strains isolated from human patients or poultry sources in Korea. However, the discrimination power of these methods for genetically closely related Salmonella Enteritidis strains is limited. The next-generation sequencing methodologies such as whole-genome sequencing (WGS) has been improved the resolution of genome analyses, such that sources of Salmonella outbreaks can be traced without using the genotyping approaches described above . Recently, isolation of MDR Salmonella has been increased from human and poultry, and these MDR Salmonella had the same antibiotic resistance phenotype . However, the genetic relationship between Salmonella Enteritidis isolated from human and poultry sources in Korea has not been investigated using WGS yet. Here, we isolated seven MDR Salmonella Enteritidis strains from poultry sources in Korea and sequenced their genomes using the Oxford Nanopore approach. In order to investigate the relationships between Salmonella strains, the whole genome sequences of the MDR isolates were compared with whole genome sequences of the previously reported Korean SalmonellaEnteritidis strains using whole genome single nucleotide polymorphism (SNP) - based phylogenetic analysis and comparative plasmid analysis.

\section{Material and Methods}

\section{Salmonella isolation from poultry sources}

During April 1-October 30, 2019, a total of 293 samples were collected and tested for Salmonella Enteritidis (230 from retail chicken meats, 31 from internal organs of chicken, and 32 from straw bedding samples from geographically seperated multiple poultry farms) were aseptically placed in a sterile plastic bag containing $400 \mathrm{~mL}$ of buffered peptone water broth (BPW, Difco, Detroit, MI, USA) and shaken for $2 \mathrm{~min}$. The rinsed material $(20 \mathrm{~mL})$ was vortex-mixed in $20 \mathrm{~mL}$ of BPW for $15 \mathrm{~s}$, and then incubated at $37^{\circ} \mathrm{C}$ for $24 \mathrm{~h}$. Incubated BPW $(100 \mu \mathrm{L})$ was vortex-mixed for $15 \mathrm{~s}$ in $10 \mathrm{~mL}$ of Rappaport-Vassiliadis broth, then incubated at $41.5^{\circ} \mathrm{C}$ for $20 \mathrm{~h}$. The presence of Salmonella spp . in the incubated Rappaport-Vassiliadis broth was analysed by PCR as described previously. Samples that yielded positive results in PCR analysis were streaked onto the Salmonella ChromoSelect agar (Sigma-Aldrich, USA), followed by incubation at $37^{\circ} \mathrm{C}$ for $24 \mathrm{~h}$. The pink 
colonies of Salmonella spp. on the agar were validated by PCR and positive colonies were stored at $-80^{\circ} \mathrm{C}$ in glycerol.

\section{Antibiotic susceptibility test}

Antibiotic susceptibility was determined using the Sensititre ${ }^{\mathrm{TM}}$ panel (KRCDC2F; Thermo Fisher Scientific, Waltham, MA, USA) with the following antibiotics: ciprofloxacin (CIP, 0.03-0.5 $\mu \mathrm{g}$ ), nalidixic acid (NAL, 2-128 $\mu \mathrm{g}$ ), imipenem (IMI, 1-8 $\mu \mathrm{g}$ ), colistin (COL, 2-16 $\mu \mathrm{g}$ ), ampicillin (AMP, 2-64 $\mu \mathrm{g}$ ), tetracycline (TET,

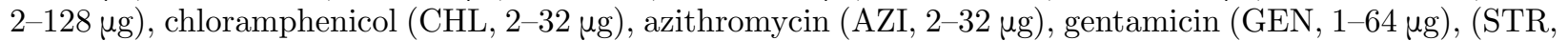

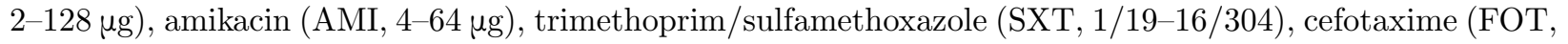
1-32 $\mu \mathrm{g}$ ), ceftriaxone (AXO, 1-32 $\mu \mathrm{g}$ ), cefoxitin (FOX, 4 - $32 \mu \mathrm{g}$ ), and ceftazidime (TAZ, 1-16 $\mu \mathrm{g}$ ), according to Clinical and Laboratory Standards Institute guidelines (Wayne, PA, USA) . Briefly, $10 \mu \mathrm{L}$ portions of Salmonella spp. strains $\left(1 \times 10^{5} \mathrm{cfu} / \mathrm{mL}\right)$ cultured overnight were thoroughly mixed with $11 \mathrm{~mL}$ of Muller Hinton Broth with $N$-Tris (hydroxymethyl) methyl-2-aminoethanesulfonic acid; $50 \mu \mathrm{L}$ portions were placed in the wells of the Sensititre ${ }^{\mathrm{TM}}$ panel. The panel was sealed with film, and the results were assessed manually after $24 \mathrm{~h}$ incubation at $37^{\circ} \mathrm{C}$. The MIC was recorded as the lowest concentration of antibiotic that inhibit visible growth shown as turbidity or deposit of cells at the bottom of a wall. Escherichia coli ATCC25922 was used as the quality control standard.

\section{Extraction and WGS of Salmonella genomic DNA}

We extracted genomic DNA from overnight cultured Salmonella spp. using the MagAttract ${ }^{\circledR}$ kit (Qiagen GmbH, Hilden, Germany) as described by the manufacturer. The purity and concentration of the extracted DNA were measured with the NanoDrop ${ }^{\mathrm{TM}}$ spectrophotometer (Thermo Fisher Scientific) and the Quantus $^{\text {TM }}$ fluorometer (Promega, Madison, WI, USA), respectively. A library was prepared for sequencing using the Native barcoding genomic DNA kits and WGS sequencing was performed using the MinION system (Oxford Nanopore Technologies Ltd., Oxford, UK) as described by the respective manufacturers. The library was loaded onto FLO-MIN106 R9.4.1 Flow Cells and sequenced for $48 \mathrm{~h}$. Data were base-called using the Albacore (Oxford Nanopore Technologies Ltd). A library prepared using the TrueSeq Nano DNA (Illumina, San Diego, CA, USA) was also sequenced using the HiSeq4000 system (Illumina, San Diego, CA, USA) for error correction of the nanopore sequencing results.

\section{Assembly, polishing, and annotation of Salmonella DNA}

Reads generated from nanopore sequencing were downsampled to generate ${ }^{\sim} 100 \times$ coverage of the Salmonella Enteritidis genome using the seqtk (https://github.com/lh3/seqtk). Downsampled reads were de novoassembled using the Flye algorithm with default parameters. The assembled sequence was polished using the Unicycler_polish with the assembled contigs and the Illumina fastq reads with default parameters. The assembled Salmonella genome was annotated using the Prokka .

\section{Data analysis}

Antibiotic resistance genes were identified using the Resfinder. SNPs between the whole genomes of the sequenced Salmonella Enteritidis strains in this study and those of the Korean SalmonellaEnteritidis strains deposited in the public database were identified and aligned with the PhaME using default parameter. The genomic sequence ofSalmonella Enteritidis P125109 strain (GenBank no. NC011294) was used as reference genome for SNP calling. A whole genome SNP tree was constructed based on 819 SNPs generated by the PhaME using the RAxML, with the General Time Reversible gamma substitution model and 100 bootstrap replicates. Plasmids were compared using the BLAST Ring Image Generator (BRIG) and the MAUVE .

\section{RESULTS AND DISCUSSION}

Ten of Salmonella Enteritidis were isolated from 293 samples. Among them, seven of Salmonella Enteritidis classified as MDR showed resistance to five antibiotic classes including Quinolones (NAL), Polymyxin (COL), Aminoglycosides (GEN), third generation cephalosporins (FOT, AXO, TAZ), Penicillins (AMP) and Tetracyclines (TET) and those with intermediate resistance to Quinolones (Table 1). All MDR isolates were 
susceptible to Carbapenems (IMI), Phenicols (CHL), Macrolides (AZI), Aminoglycoside (STR, AMI) and third generation cephalosporins (FOX) (Table 1).

Genome assembly of all the sequenced Salmonella Enteritidis strains generated 2 circular contigs consisted of a chromosome and a plasmid. The size of chromosome ranged from 4,678,918 to 4,68,786bp with a GC content of $52.2 \%$. Six of the seven isolates had a plasmid size of 110,273 with a GC content of $51.7 \%$ and Z0719SL0013 had a plasmid size of 109,445 with a GC content of $51.7 \%$. All sequence files are available from NCBI under BioProject PRJNA658425.

All of the sequenced Salmonella Enteritidis strains harboured antibiotic resistance genes and chromosomal mutations that coincided with antibiotic resistance phenotypes (Table 1). The antibiotic resistance gene aac (6')-laa_1 (aminoglycoside) was detected on the chromosome, whereas the aac(3')-lld_1 (gentamicin),blaCTX-M-15 (cephalosporin), and tet(A) (tetracycline) were detected on the plasmid found in all of the MDR isolates. Chromosomal mutations in the gyrA (quinolone) and phoQ(colistin) were also found (Table1).

The whole genome SNP tree generated by the RAxML based on results of the PhaME analysis showed that all of the isolated SalmonellaEnteritidis strains were genetically close with a median pair-wise distance of 8 (range 1-16) SNPs (Figure 1). Further, all of the sequenced isolates were clustered together with the FORC_019 strain, isolated from human blood with salmonellosis, showing monophyletic relationship with bootstrap support of 100. The median pair-wise SNP distance of the FORC_019 strain from the other isolates was 11.2 (range 10-16). A pairwise SNP distance of [?]20 and monophyletic relationship with a bootstrap support value $>0.9$ indicated that these strains originated from the same genetic ancestor . Results of whole genome SNP analysis betweenSalmonella Enteritidis strains isolated from human and poultry sources were consistent with those of the previous PFGE and MLVA studies .

All of the MDR strains isolated in this study harboured an $110 \mathrm{~kb}$ plasmid (pSE-VMDR) with the IncFIB and IncFII replicons and 51.7\% GC content, including 146 ORFs. Six of the seven isolates had a plasmid with the same genomic structure. Compared with other plasmids, an inverted genomic region of $\sim 52 \mathrm{~kb}$ in size was detected in the pZ0719SL0013 (Figure 2a). An inverted genomic region $~ 59.5 \mathrm{~kb}$ in size like that in the pZ0719SL0013 was found in the pFORC_019. The inverted region of the pFORC_019 contained a $7.5 \mathrm{~kb}$ insertion sequence comprising a Tn6029 remnant and Tn4352, which encoded multiple antibiotic resistance genes, i.e.,sul2-str $A$-strB and $A P H(3)$, respectively.

BLAST comparison between the pSE-VMDR and the pFORC_019 revealed 99\% of sequence identity and $93.5 \%$ of coverage (Figure $2 \mathrm{~b}$ ). This comparison also revealed that the pSE-VMDR was a novel fusion plasmid consisting of the pSEJ and the FORC_038 chromosome, which contains genes encoding virulence factors and genes encoding antibiotic resistance proteins, respectively. Salmonella Enteritidis contains a 59 $\mathrm{kb}$ serotype specific virulence plasmid, pSENV . Studies of clinicalSalmonella Enteritidis isolates have shown that the antibiotic resistance gene has been introduced into the virulence plasmid. Nucleotide sequence identity of the antibiotic resistance region in the pSE-VMDR was $100 \%$ with that of the chromosome of the FORC_038 strain of Salmonella Virchow isolated from raw chicken meat in Korea except for a 7.5 kb insertion sequence in pFORC_019. SalmonellaEnteritidis, Salmonella Montevideo, and Salmonella Virchow are the most prevalent serotypes of Salmonella entericaisolated from chicken meats and chicken slaughterhouses in Korea . Clonal dissemination of Salmonella Virchow carrying the CTX-M-15 gene may have occurred in contaminated food in Korea . SalmonellaEnteritidis strains isolated from chicken and human stools in Korea during 2009 carried a $95 \mathrm{~kb}$ of conjugative plasmid harbouring the CTX-M-15 gene . The antibiotic resistance phenotype, plasmid size, and plasmid replicon type in the seven MDR isolates studied herein were consistent with those of the 2009 isolates. The existence of the ISEcp1 upstream from the CTX-M-15 was also consistent.

In conclusion, Salmonella Enteritidis strains that can infect human and poultry have been circulating through clonal or horizontal transmission for a long time in Korea. Since we showed that the antibiotic resistance genes can be transmitted via plasmids betweenSalmonella enterica strains in an intraspecies manner, antibiotics 
against which this plasmid confers resistance have been specifically avoided at poultry farms to prevent the further spread of such plasmids among Salmonella Enteritidis strains. This is critically important to prevent the prevalence of MDR Salmonellafrom increasing

\section{Ethical statement}

The authors confirm that the ethical policies of the journal, as noted on the journal's author guidelines page, have been adhered to. No ethical approval was required as this is a review article with no original research data.

\section{Conflict of interest statement}

The authors have nothing to disclose

\section{REFERENCES}

Table 1. Antibiotic resistance profiles of the MDR strains ofSalmonella Enteritidis isolated in this study

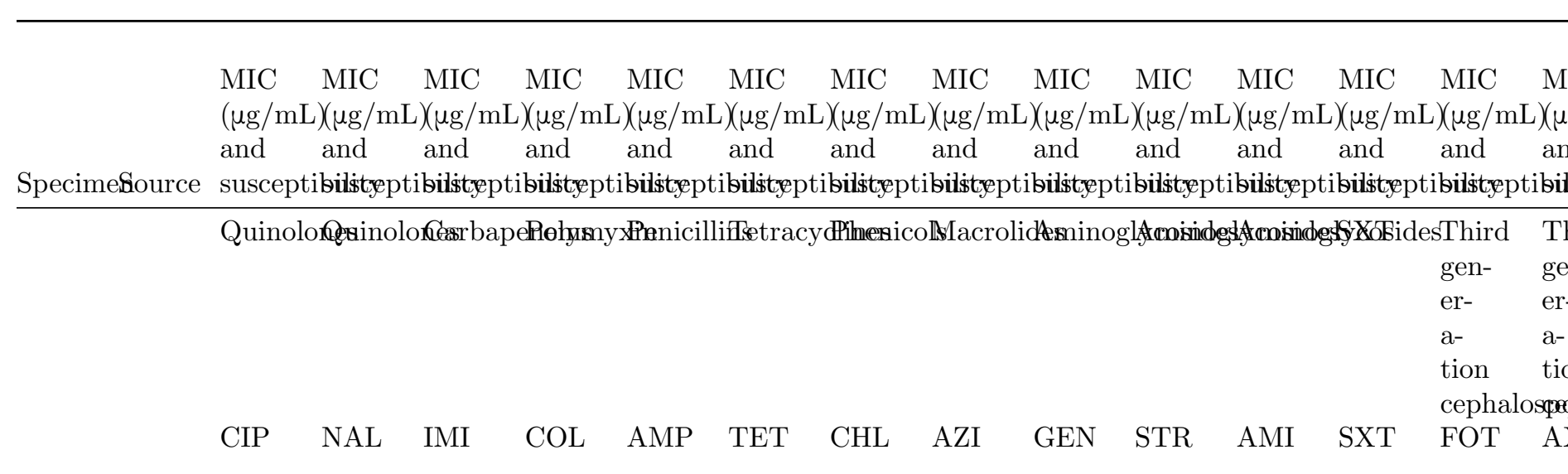


MIC MIC MIC MIC MIC MIC MIC MIC MIC MIC $\quad$ MIC $\quad$ MIC $\quad$ MIC $\quad$ M $(\mu \mathrm{g} / \mathrm{mL})(\mu \mathrm{g} / \mathrm{mL})(\mu \mathrm{g} / \mathrm{mL})(\mu \mathrm{g} / \mathrm{mL})(\mu \mathrm{g} / \mathrm{mL})(\mu \mathrm{g} / \mathrm{mL})(\mu \mathrm{g} / \mathrm{mL})(\mu \mathrm{g} / \mathrm{mL})(\mu \mathrm{g} / \mathrm{mL})(\mu \mathrm{g} / \mathrm{mL})(\mu \mathrm{g} / \mathrm{mL})(\mu \mathrm{g} / \mathrm{mL})(\mu \mathrm{g} / \mathrm{mL})(\mu$ and and and and and and and and and and and and and an Specimefource susceptibilstyptibilisteptibilistyptibilistyptibilistyptibilistyptibilistyptibilistyptibilityeptibilityeptibilityeptibilityeptibil

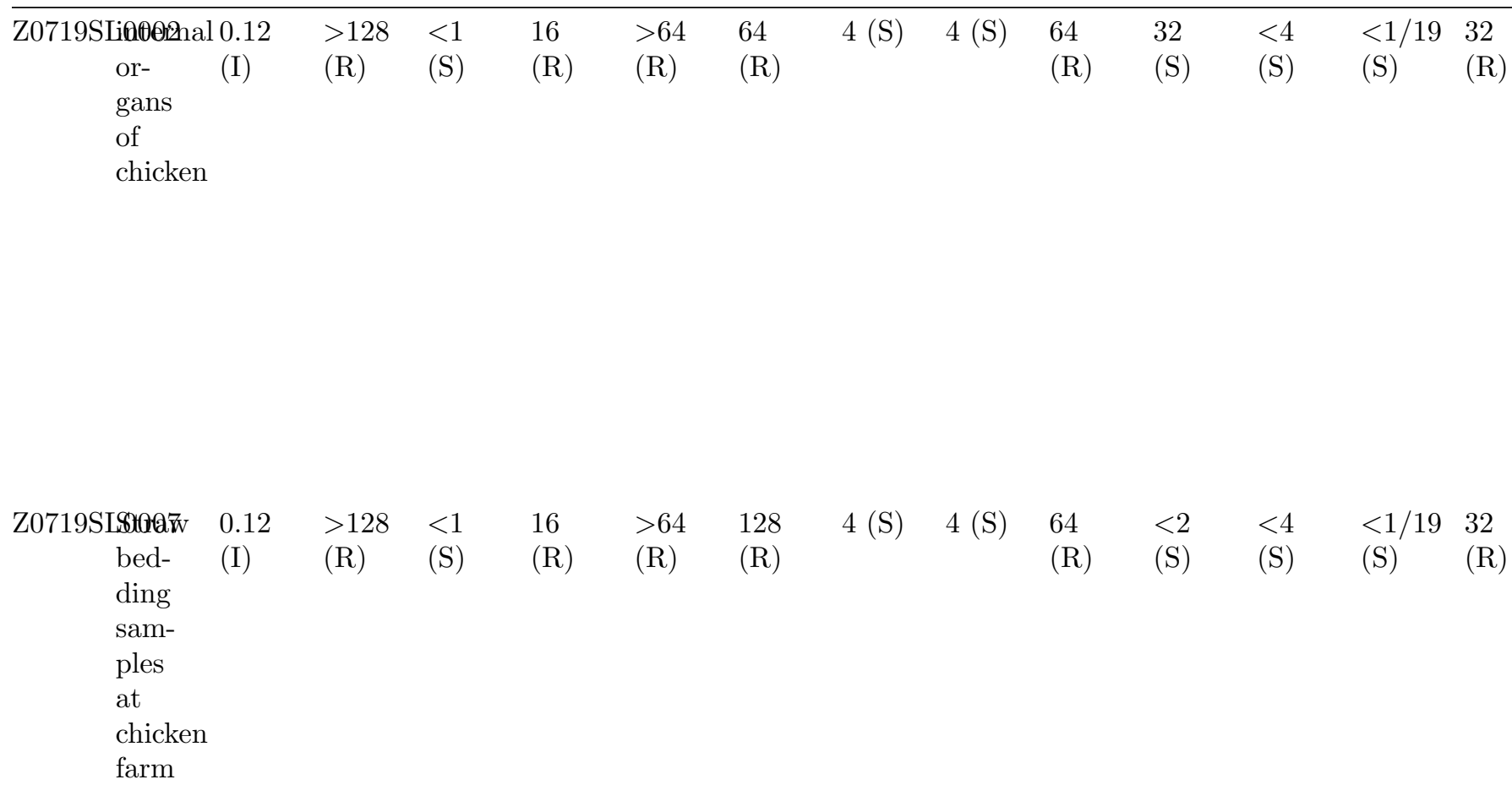

$8(\mathrm{R}) \quad>64 \quad 64$

(R) $\quad(\mathrm{R})$
$4(\mathrm{~S})$

meat

$(\mathrm{R})$

$(\mathrm{S})$

$4(\mathrm{~S}) \quad 64$

(R)

4 (S)

$<4$

(S)

$<1 / 1$

$<1 / 19>32$

(R) 
MIC MIC MIC MIC MIC MIC MIC MIC MIC MIC MIC MIC MIC $\quad$ M $(\mu \mathrm{g} / \mathrm{mL})(\mu \mathrm{g} / \mathrm{mL})(\mu \mathrm{g} / \mathrm{mL})(\mu \mathrm{g} / \mathrm{mL})(\mu \mathrm{g} / \mathrm{mL})(\mu \mathrm{g} / \mathrm{mL})(\mu \mathrm{g} / \mathrm{mL})(\mu \mathrm{g} / \mathrm{mL})(\mu \mathrm{g} / \mathrm{mL})(\mu \mathrm{g} / \mathrm{mL})(\mu \mathrm{g} / \mathrm{mL})(\mu \mathrm{g} / \mathrm{mL})(\mu \mathrm{g} / \mathrm{mL})(\mu$ and and and and and and and and and and and and and an Specimefource susceptibilstyptibilisteptibilistyptibilistyptibilistyptibilistyptibilistyptibilistyptibilityeptibilityeptibilityeptibilityeptibil

\begin{tabular}{clllllllllllll}
\hline $\begin{array}{r}\text { Z0719SLObilken } 0.25 \\
\text { meat }\end{array}$ & $>128$ & $<1$ & 16 & $>64$ & 64 & $8(\mathrm{~S})$ & $4(\mathrm{~S})$ & 64 & $4(\mathrm{~S})$ & $<4$ & $<1 / 19$ & $>32$ & $>$ \\
$(\mathrm{R})$ & $(\mathrm{S})$ & $(\mathrm{R})$ & $(\mathrm{R})$ & $(\mathrm{R})$ & & & $(\mathrm{R})$ & & $(\mathrm{S})$ & $(\mathrm{S})$ & $(\mathrm{R})$ & $(\mathrm{R}$
\end{tabular}

9Sintedinal $0.25=128$

or-

(I) $\quad(\mathrm{R}) \quad(\mathrm{S})$

$8(\mathrm{R}) \quad \begin{array}{ll}>64 & 64 \\ (\mathrm{R}) & (\mathrm{R})\end{array}$

$4(\mathrm{~S})$

$4(\mathrm{~S}) \quad 64$

$8(\mathrm{~S})$

$<4$
$(\mathrm{~S})$

$<1 / 19>32$

gans

of

chicken

Z0719SLOBicken $0.25>128<1$

meat

(I)

(R) (S)
$4(\mathrm{R}) \quad>64$

(R)

128

$4(\mathrm{~S})$

(R)

$4(\mathrm{~S}) \quad 64$

(R)

$4(\mathrm{~S})$

$<4$

(S)

(S)

$<1 / 19 \quad 32$

(R) 


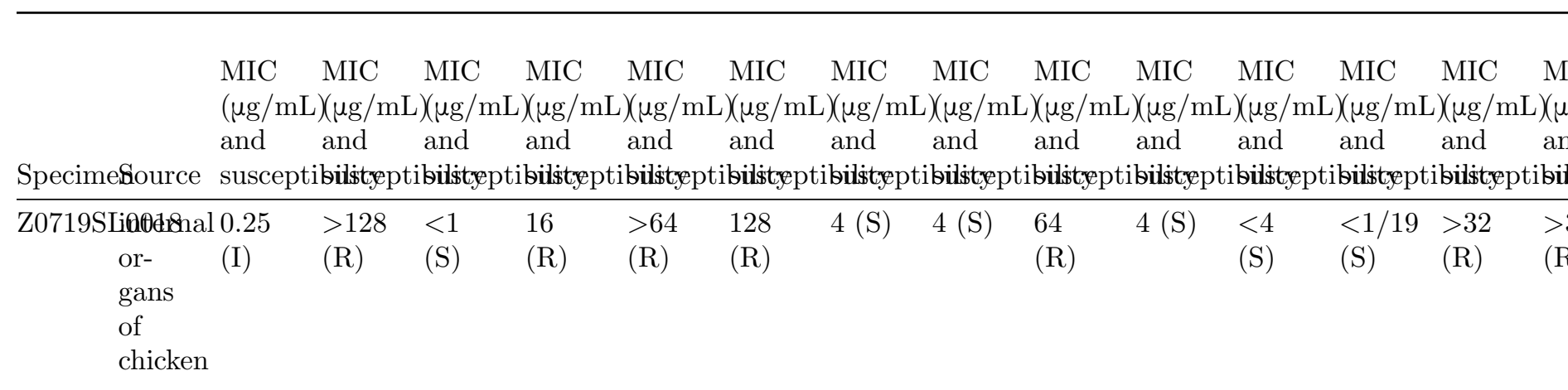

AMI, amikacin; AMP, ampicillin; AXO, ceftriaxone; AZI, azithromycin; CHL, chloramphenicol; CIP, ciprofloxacin; COL, colistin; FOT, cefotaxime; FOX, cefoxitin; GEN, gentamicin; I, intermediate; IMI, imipenem; NAL, nalidixic acid; R, Resistant; S, susceptible; STR, streptomycin; SXT, trimethoprim/sulfamethoxazole; TAZ, ceftazidime; TET, tetracycline.

\section{FIGURE LEGENDS}

Figure 1. Maximum-likelihood tree of Korean SalmonellaEnteritidis strains based on whole-genome SNP analysis visualized using interactive Tree Of Life version 5 (iTOLv5) (htttps://iltol.embl.de/). The tree was rooted at midpoint. Pairwise SNP distance (1 to 91 SNPs) are indicated by heat map. Colours in the heat map indicate the numbers of pairwise SNP distance between isolates. The scale bar measures the numbers of substitutions per site. Only boot strap support greater than 50 is shown.

Figure 2 . Comparative plasmid analysis of Salmonella Enteritidis plasmids.(A) MAUVE alignment of seven plasmids of Salmonella Enteritidis and pFORC_019 (GenBank no. CP012397) (B) Wholegenome sequence of plasmid from pFORC_019 compared with seven plasmid of SalmonellaEnteritidis, virulence plasmid pSEJ (GenBank no. CP008927) and chromosome of FORC_038 (GenBank no. 015574). Antibiotic resistance genes are marked in red on the outer ring. IS elements are marked in black on the outer ring. Virulence gene are marked in blue on the outer ring. 
figures/Figure1/Figure1-eps-converted-to.pdf 
figures/Figure2/Figure2-eps-converted-to.pdf 